\title{
Stability criteria for nonlinear Volterra integro-dynamic matrix Sylvester systems on measure chains
}

\author{
Sreenivasulu Ayyalappagari1 * (D) and Venkata Appa Rao Bhogapurapu ${ }^{2}$
}

\section{"Correspondence:}

sreenu5408@gmail.com

'Department of Mathematics,

Koneru Lakshmaiah Education

Foundation and Malla Reddy

Institute of Technology and Science,

Dhulapally, Secunderabad, 500100,

Telangana, India

Full list of author information is

available at the end of the article

\begin{abstract}
In this paper, we establish sufficient conditions for various stability aspects of a nonlinear Volterra integro-dynamic matrix Sylvester system on time scales. We convert the nonlinear Volterra integro-dynamic matrix Sylvester system on time scale to an equivalent nonlinear Volterra integro-dynamic system on time scale using vectorization operator. Sufficient conditions are obtained to this system for stability, asymptotic stability, exponential stability, and strong stability. The obtained results include various stability aspects of the matrix Sylvester systems in continuous and discrete models.
\end{abstract}

Keywords: Nonlinear Volterra integro-dynamic; Stability; Timescales

\section{Introduction}

The applications of differential equations in science and engineering problems are well known. Difference equations are the discrete counterpart of differential equations. The applicability of difference equations is gaining an important role in computer science, control theory, image processing, digital filter design, numerical analysis, and finite element techniques. Agarwal [1] presented several theoretical methods and applications of difference equations. The $\Psi$-stability for difference equations is studied in [2].

In real life, applications are simple and efficient for integral equations. The existence of solutions for nonlinear quadratic integral equations was obtained in [3]. The solution of Fredholm integral equations via fixed point on extending b-metric spaces was introduced in [4]. The solutions of Volterra integral equations were obtained using numerical scheme in d-metric spaces [5], fixed point theory [6], and hybrid contractions [7]. Recently, fractional differential equations have been applied in many areas of science and engineering. Many authors [8-11] studied different types of factional differential equations. Kim [12] studied semi-linear problems with a non-symmetric linear part and a nonlinear part of monotone type in real Hilbert spaces. Nguyen Duc Phuonga [13] proved that the regularized solution satisfies the conditions of a well-posed problem in the sense of Hadamard and also used the modified quasi-boundary method to deal with the inverse source problem, a well-posed method.

(c) The Author(s) 2021. This article is licensed under a Creative Commons Attribution 4.0 International License, which permits use, sharing, adaptation, distribution and reproduction in any medium or format, as long as you give appropriate credit to the original author(s) and the source, provide a link to the Creative Commons licence, and indicate if changes were made. The images or other third party material in this article are included in the article's Creative Commons licence, unless indicated otherwise in a credit line to the material. If material is not included in the article's Creative Commons licence and your intended use is not permitted by statutory regulation or exceeds the permitted use, you will need to obtain permission directly from the copyright holder. To view a copy of this licence, visit http://creativecommons.org/licenses/by/4.0/. 
Time scales theory was introduced by Hilger $[14,15]$ to unify discrete and continuous cases in a well-organized structure. For an excellent introduction to the calculus and dynamic equations on time scales, one can refer to [1, 16-18]. In [19], the authors presented basic qualitative and quantitative results for solutions to nonlinear dynamic equations on time scales with applications to economic modeling. The authors $[20,21]$ studied stability of dynamic equations on time scales. The solution of Volterra integro-dynamic equations on time scales using variation of parameter was obtained in [22] and the qualitative and quantitative results were obtained in [23]. In [24], authors established the existence of $\Psi$-bounded solutions for a system of linear dynamic equations on time scales. Recently, Agarwal [25] described the connection between the F-contraction mappings on metriclike spaces to integral equations on time scales.

The matrix differential systems are generalizations of a system of differential equations. The fundamental matrix of a system consists of linearly independent solutions. The transition matrix of a system is obtained from fundamental matrix. Dacunha [26] studied transition matrix and generalized matrix exponential via the Peano-Baker series. Murty et al. [27-30] studied matrix Lyapunov systems. The controllability for a fuzzy dynamical matrix Lyapunov system was studied in [31]. In [32], authors established the necessary and sufficient conditions for controllability and observability of the Sylvester matrix dynamical system on time scales. In this paper, we deal with a nonlinear Volterra integro-dynamic matrix Sylvester system on time scales and establish the conditions for stability, asymptotic stability, exponential stability, and strong stability.

$$
\left\{\begin{aligned}
X^{\Delta}(t)= & A(t) X(t)+X(t) B(t)+\mu(t) A(t) X(t) B(t) \\
& +\int_{t_{0}}^{t}\left(L_{1}(t, X(s)) X(s)+X(s) L_{2}(t, X(s))\right) \Delta s, \\
X\left(t_{0}\right)= & X_{0},
\end{aligned}\right.
$$

where $\mathrm{X}(\mathrm{t})$ is an $n \times n$ matrix, Here, $A(t), B(t), L_{1}(t)$, and $L_{2}(t)$ are $n \times n, n \times n . n \times n$ and $n \times m$ are rd-continuous matrices respectively, $X^{\Delta}(t)$ is the generalized delta derivative of $\mathrm{X}$, and $\mu(t)$ is a graininess function.

\section{Preliminaries}

Definition 2.1 ([16]) A time scale $\mathbb{T}$ is a closed subset of $\mathbb{R}$. By an interval we mean the intersection of the given interval with a time scale. For $t<\sup \mathbb{T}$ and $r>\inf \mathbb{T}$, define the forward jump operator $\sigma$ and backward jump operator $\rho$ respectively by $\sigma(t)=\inf \{s \in$ $T: s>t\} \in \mathbb{T}, \rho(t)=\sup \{s \in T: s<r\} \in \mathbb{T}, \forall t, r \in \mathbb{T}$ if $\sigma(t)=t$, $\mathrm{t}$ is said to be right-dense (otherwise $\mathrm{t}$ is said to be right-scattered) and $\rho(r)=r$, $\mathrm{r}$ is said to be left-dense (otherwise $\mathrm{r}$ is said to be left-scattered). The graininess function $\mu(t): \mathbb{T} \rightarrow[0, \infty)$ is defined by $\mu(t)=$ $\sigma(t)-t$.

Definition 2.2 ([16]) For $x: \mathbb{T} \rightarrow \mathbb{R}$ and $t \in T$ (if $t=\sup \mathbb{T}$, assume that $\mathrm{t}$ is not leftscattered), define the delta derivative of $\mathrm{x}(\mathrm{t})$, represented by $x^{\Delta}(\tau)$, to be a number, with the property that, for any $\epsilon>0, \exists$ in the neighborhood $U$ of $t$ such that

$$
\left|[x(\sigma(t))-x(s)]-x^{\Delta}(t)[\sigma(\tau)-s]\right| \leq \varepsilon|\sigma(t)-s|, \quad \forall s \in U
$$

If $\mathrm{x}$ is delta differentiable for every $t \in T$, we say that $x: \mathbb{T} \rightarrow \mathbb{R}$ is delta differentiable on $\mathrm{T}$. 
Definition 2.3 ([16]) A function $h$ is known as rd-continuous provided that it is continuous at right-dense points in $T$, and finite limit at left-dense points, and the set of rdcontinuous functions is denoted by $C_{r d}(\mathbb{T}, \mathbb{R})$. The set of functions $C_{r d}^{1}(\mathbb{T}, \mathbb{R})$ includes the functions $\mathrm{h}$ whose derivative is in $C_{r d}(\mathbb{T}, \mathbb{R})$.

Definition 2.4 ([16]) For $a, t \in \mathbb{T}$ and a function $h \in C_{r d}(\mathbb{T}, \mathbb{R})$, delta-integral is defined to be

$$
\int_{a}^{t} h(s) \Delta s=H(t)-H(a)
$$

where $H \in C_{r d}^{1}(\mathbb{T}, \mathbb{R})$ is anti-derivative of h, i.e., $H^{\Delta}(t)=h$.

Theorem 2.1 ([16]) Assume that $h: \mathbb{T} \rightarrow \mathbb{R}$ is a function, and let $t \in \mathbb{T}^{k}$. Then we have the following:

i. If $t$ is right-dense, then $h$ is differentiable at $t$ iff the limit $\lim _{t \rightarrow s} \frac{h(t)-h(s)}{t-s}$ exists as a finite number. In this case $h^{\Delta}(t)=\lim _{t \rightarrow s} \frac{h(t)-h(s)}{t-s}$.

ii. If $h$ is differentiable at $t$, then $h(\sigma(t))=h(t)+\mu h^{\Delta}(t)$.

iii. If $h$ is continuous at $t$ and $t$ is right-scattered, then $f$ is differentiable at $t$ with $h^{\Delta}(t)=\frac{h(t)-h(s)}{\mu(t)}$.

iv. If $h$ is continuous at $t$, then $f$ is differentiable at $t$.

Definition 2.5 ([32]) Let two right-dense continuous matrices be $A_{1}$ and $A_{2}$ on $\mathbb{T}$, on time scales $\mathbb{T}$. Therefore,

$$
A_{1}^{\Delta}(t) \otimes A_{2}(t)+A_{1}(\sigma(t)) \otimes A_{2}^{\Delta}(t)=\left(A_{1} \otimes A_{2}\right)^{\Delta}(t)
$$

Now, we are applying the Vec operator to the delta-differentiable Volterra integrodynamic matrix Sylvester system (1) by using Kronecker product (KP) properties, we obtain

$$
\left\{\begin{array}{l}
z^{\Delta}(t)=P(t) z(t)+\int_{0}^{t} G(t, s, z(s)) \Delta s, \\
z\left(t_{0}\right)=z_{0},
\end{array}\right.
$$

which is piecewise continuous on $\Omega:=\left\{(t, s) \in \mathbb{T}_{0} \times \mathbb{T}_{0}: t_{0} \leq s \leq t<\infty\right\}$, and $P(t)=\left[\left(B^{*} \otimes\right.\right.$ $\left.I)+(I \otimes A)+\mu(t)\left(B^{*} \times A\right)\right]$ of order $n^{2} \times n^{2}, G(t, s, z(s))=\left[\left(L_{2}^{*} \otimes I\right)+\left(I \otimes L_{1}\right)\right]$ is an $n^{2} \times n^{2}$ matrix function. $z(t)=\operatorname{Vec} X(t)$.

Definition 2.6 ([16]) For $P \in \mathcal{R}$, the generalized exponential function is defined as

$$
e_{P}(t, s)=\exp \left(\int_{s}^{t} \xi_{\mu}(t)(P(\tau)) \Delta \tau\right), \quad t, s \in \mathbb{T}
$$

where $\xi_{\mu}(t)(P(\tau))$ is the cylinder transformation given by

$$
\xi_{\mu}(t)(P(\tau))= \begin{cases}\frac{\log (1+\mu(\tau) P)}{\mu(\tau)} & \text { if } \mu(\tau) \neq 0, \\ P & \text { if } \mu(\tau) \neq 0 .\end{cases}
$$


Let the complete metric space with the distance (metric) $\mathrm{d}$ be defined by $d\left(\left(u_{1}, v_{1}\right)\right.$, $\left.\left(u_{2}, v_{2}\right)\right)=\sqrt{\left(v_{1}-v_{2}\right)^{2}+\left(u_{1}-u_{2}\right)^{2}}, \forall\left(u_{1}, v_{1}\right),\left(u_{2}, v_{2}\right) \in \mathbb{T}_{1} \times \mathbb{T}_{2}$.

Here, $\mathbb{T}_{1} \times \mathbb{T}_{2}=\left\{(u, v): u \in \mathbb{T}_{1}, v \in \mathbb{T}_{2}\right\}$ are two given time scales.

A function $f: \mathbb{T}_{1} \times \mathbb{T}_{2} \rightarrow \mathbb{R}$ is called continuous at $(u, v) \in \mathbb{T}_{1} \times \mathbb{T}_{2}, \forall \epsilon>0, \exists \delta>0$ such that $\left\|f(u, v)-f\left(u_{0}, v_{0}\right)\right\|<\epsilon, \forall\left(u_{0}, v_{0}\right) \in \mathbb{T}_{1} \times \mathbb{T}_{2}$ satisfying $d\left((u, v),\left(u_{0}, v_{0}\right)<\delta\right)$.

\section{Lemma 2.1 ([18])}

i. If $\psi$ is rd-continuous and nonnegative, thus

$$
1+\int_{s}^{t} \psi(u) \Delta u \leq e_{\psi}(t, s) \leq \exp \left(\int_{s}^{t} \psi(u) \Delta u\right) \quad \forall t \geq s .
$$

ii. For a nonnegative $\psi$ with $-\psi \in \mathcal{R}^{+}$, it becomes

$$
1-\int_{s}^{t} \psi(u) \Delta u \leq e_{-\psi}(t, s) \leq \exp \left(-\int_{s}^{t} \psi(u) \Delta u\right) \quad \forall t \geq s .
$$

Theorem 2.2 ([26]) Let $f(u, v)$ be a real finite-valued function whose domain is the cartesian product $S_{1} \times S_{2}$. Suppose that $f(u, v)$ is continuous in $u$ at $u=a$ for all $v$ in $S_{2}$, and continuous in $v$ at $v=b$ uniformly for $u$ in $S_{1}$, then $f(u, v)$ is continuous in $(u, v)$ at $(a, b)$.

Let $r d$-continuous functions $f(u, v)$ on $\mathbb{T}_{1} \times \mathbb{T}_{2}$ have the following properties:

i. $f$ is $r d$-continuous in $v$ for fixed $u$;

ii. $f$ is $r d$-continuous in $u$ for fixed $v$;

iii. If $u_{0}$ and $v_{0}$ are both left-dense, thus the limit of $f(u, v)$ exists (finite) as $(u, v)$ approaches $\left(u_{0}, v_{0}\right)$ along any path in $(u, v) \mathbb{T}_{1} \times \mathbb{T}_{2}: u<u_{0}, v<v_{0}$;

iv. If $\left(u_{0}, v_{0}\right) \in \mathbb{T}_{1} \times \mathbb{T}_{2}$ with $u_{0}$ maximal or right-dense and $v_{0}$ maximal or right-dense. Thus, $f$ is continuous at $\left(u_{0}, v_{0}\right)$.

\section{Stability}

In this section, when we say the zero solution of (2) we mean the zero solution of (2) with $z_{0}=0$.

Definition 3.1 The zero solution of (2) is stable. If for every $\epsilon>0$ there exists $\delta>0$ such that, for any solution $\mathrm{z}(\mathrm{t})$ of (2), the inequality $\left\|z_{0}\right\|<\delta \Longrightarrow\|z\|<\epsilon \forall t \in \mathrm{T}_{0}$.

We assume that the zero solution of the following system

$$
\left\{\begin{array}{l}
x^{\Delta}(t)=P(t) x(t) \\
x\left(t_{0}\right)=x_{0}
\end{array}\right.
$$

is stable. From Theorem 2.1 [20] we suppose that there exists $\eta>0$ such that

$$
\left\|\psi_{p}(t . s)\right\| \leq \eta, \quad \forall s \in\left[t_{0}, t\right]_{\mathbb{T}}
$$

where $\psi_{p}(t . s)$ is a fundamental matrix of (3). 
Next, we impose a condition on $G(t, s, z)$ for proving that the zero solution of (2) is stable. We assume the following condition:

$H 1: \exists \alpha>0$ so that $\|G(t, s, z)\| \leq Q(t, s)\|z\|$ with $Q(t, s)$ rd-continuous for $s \in\left[t_{0}, t\right]_{\mathbb{T}}$ and $\|z\|<\alpha$.

Theorem 3.1 Assume that equation (4) and (H1) are satisfied, and there exists a positive constant $L>0$ such that

$$
\int_{t_{0}}^{\infty} \int_{t_{0}}^{s} Q(s, u) \Delta s \Delta u<L
$$

Then the zero solution of (2) is stable.

Proof . For any $0<\epsilon<\alpha$, let $\delta(\epsilon)<\frac{\epsilon}{\eta e^{\eta L}}$ and $\|z(t)\|<\delta(\epsilon)$.

Assume that there exists $t_{1} \in \mathbb{T}_{0}$ such that $\left\|z\left(t_{0}\right)\right\|=\epsilon$ and $\left\|z\left(t_{0}\right)\right\|<\epsilon$ on $\left[t_{0}, t_{1}\right)_{\mathbb{T}}$. By using the variation of parameters formula, we get

$$
\begin{aligned}
\|z(t)\| & <\left\|\psi_{P}\left(t, t_{0}\right)\right\|\left\|z_{0}\right\|+\int_{t_{0}}^{t}\left\|\psi_{P}(t, \sigma(s))\right\| \int_{t_{0}}^{s} Q(s, u)\|z(u)\| \Delta u \Delta s \\
& \leq \eta \delta(\epsilon)+\eta \int_{t_{0}}^{t} \int_{t_{0}}^{s} Q(s, u)\|z(u)\| \Delta u \Delta s
\end{aligned}
$$

for $t \in\left[t_{0}, t_{1}\right)_{\mathbb{T}}$.

Let $r(t)=\sup _{s \in\left[t_{0}, t_{1}\right)_{\mathbb{T}}}\|z(s)\|$, and according to

$$
r(t) \leq \eta \delta(\epsilon)+\eta \int_{t_{0}}^{t} \int_{t_{0}}^{s} Q(s, u) r(u) \Delta u \Delta,
$$

from Gronwal's inequality ([17], Theorem 6.4) and Lemma 2.1, we get

$$
\begin{aligned}
\|z(t)\| & \leq r(t) \\
& \leq \eta \delta(\epsilon) \exp \left(\int_{t_{0}}^{t} \log \left(\frac{1+\mu(s) \eta \int_{t_{0}}^{s} Q(s, u) \Delta u}{\mu(s)}\right) \Delta s\right) \\
& \leq \eta \delta(\epsilon) \exp \left(\int_{t_{0}}^{t} \int_{t_{0}}^{s} \eta Q(s, u) \Delta u \Delta s\right) \\
& \leq \eta \delta(\epsilon) e^{\eta L}<\epsilon, \quad \forall t \in\left[t_{0}, t_{1}\right)_{\mathbb{T}} .
\end{aligned}
$$

Thus, $\left\|z\left(t_{1}\right)\right\|<\epsilon$. Therefore, it is a contradiction. Then the zero solution of (2) is stable.

In place of (H1) assume that

$\hat{H} 1:\|G(t, s, z)\| \leq Q(t, s)\|z\|$ with $Q(t, s)$ rd-continuous for $s \in\left[t_{0}, t\right]_{\mathbb{T}}$ and $\|z\| \in \mathbb{R}^{n^{2}}$. Corollary. If equations (4), (5) and ( $\hat{H} 1)$ are satisfied, then the solutions of system (3) are bounded. 


\section{Asymptotic stability}

Suppose that there exists a constant $\beta>0$ such that

$$
\int_{t_{0}}^{t}\left\|\psi_{P}(t, \sigma(s))\right\| \Delta s<\beta
$$

for all $t \in \mathbb{T}_{0}$ with $t \geq \sigma\left(t_{0}\right)$ (which is similar to the results of [20]).

The fundamental matrix $\psi_{P}$ has the following property:

$$
\psi_{P}(t, \sigma(s)) \rightarrow 0 \quad \text { as } t \rightarrow \infty
$$

Definition 4.1 The zero solution of (2) is asymptotically stable. If it is stable and attractive (i.e., if for any solution $\mathrm{z}(\mathrm{t})$ of (3) there exists $\delta_{0} \geq 0$ such that $\left\|z_{0}\right\|<\delta_{0} \Longrightarrow\|z\| \rightarrow 0$ as $t \rightarrow \infty)$.

Theorem 4.1 Assume that condition (H1) and (6) are satisfied and

$$
\sup _{t \in \mathrm{T}_{0}} \int_{t_{0}}^{t} Q(t, s) \Delta s<\frac{1}{\beta}
$$

Moreover, assume that

$$
\lim _{s \rightarrow \infty} \int_{t_{0}}^{t} Q(t, s) \Delta s=0, \quad \forall t \in \mathbb{T}_{0} .
$$

Then the zero solution of (2) is asymptotically stable.

Proof . First, we have to prove the stability of the zero solution of (2). From (8), there exists a positive constant $\gamma$ such that

$$
0<\gamma<\frac{1}{\beta} \quad \text { and } \quad \sup _{t \in \mathbb{T}_{0}} \int_{t_{0}}^{t} Q(t, s) \Delta s \leq \gamma .
$$

From (7), there exists a positive constant $M$ such that

$$
\left\|\psi_{P}\left(t, t_{0}\right)\right\| \leq M, \quad \forall t \in \mathbb{T}_{0}
$$

For any $0<\epsilon<\alpha$ and $t_{0}$, let $\delta(\epsilon)<\min \left\{\frac{(1-\gamma \beta) \epsilon}{M}, \epsilon\right\}$.

Consider the solution $\mathrm{z}(\mathrm{t})$ of (2) such that $\left\|z_{0}\right\|<\delta$. Assume that there exists $t_{1} \in \mathbb{T}_{0}$ such that $\left\|z\left(t_{1}\right)\right\|=\epsilon$ and $\|z(t)\|<\epsilon$ on $\left[t_{0}, t\right]_{\mathbb{T}_{0}}$. By using the variation of parameters formula [17], we get

$$
\begin{aligned}
\|z(t)\| & \leq\left\|\psi_{P}\left(t, t_{0}\right)\right\|\left\|z_{0}\right\|+\int_{t_{0}}^{t}\left\|\psi_{P}(t, \sigma(s))\right\| \int_{t_{0}}^{s} Q(s, u)\|z(u)\| \Delta u \Delta s \\
& <N \delta+\epsilon \int_{t_{0}}^{t}\left\|\psi_{P}(t, \sigma(s))\right\| \int_{t_{0}}^{s} Q(s, u) \Delta u \Delta s \\
& <(1-\gamma \beta) \epsilon+\epsilon \beta \gamma=\epsilon, \quad \forall t \in\left[t_{0}, t\right]_{\mathbb{T}} .
\end{aligned}
$$


Therefore, $\left\|z\left(t_{1}\right)\right\|<\epsilon$, hence, it is a contradiction. Thus, the zero solution of (2) is stable.

Next, we prove that the zero solution of (2) is attractive. Let $\epsilon=1, \exists \delta_{0}<\delta(1)<1$ such that $\left\|z_{0}\right\|<\delta_{0}$ indicates that

$$
\|z(t)\|<\min (\alpha, 1), \quad \forall t \in \mathbb{T}_{0} .
$$

Assume that there exists $z_{0}$ with $\left\|z_{0}\right\|<\delta_{0}$ such that the solution $\mathrm{z}(\mathrm{t})$ of (2) satisfies

$$
\limsup _{t \rightarrow \infty}\|z(t)\|=\lambda>0
$$

From (10), $\gamma \beta<1$, and there exists a constant $\theta$ such that $\gamma \beta<\theta<1$. From (13) there exists $t_{1} \in \mathbb{T}_{0}$ such that

$$
\|z(u)\| \leq \frac{\lambda}{\theta}, \quad \forall u \in\left[t_{1}, \infty\right)_{\mathbb{T}},
$$

and from (9) there exists $T \in\left[t_{1}, \infty\right)_{\mathbb{T}}$ such that

$$
\int_{t_{0}}^{t_{1}} Q(s, u) \Delta u<\frac{(\theta-\gamma \beta) \lambda}{2 \theta \beta}, \quad \forall s \in[T, \infty)_{\mathbb{T}} .
$$

Then

$$
\begin{aligned}
\|z(t)\| \leq & \left\|\psi_{P}\left(t, t_{0}\right)\right\| \delta_{0}+\int_{t_{0}}^{t}\left\|\psi_{P}(t, \sigma(s))\right\| \int_{t_{0}}^{s} Q(s, u)\|z(u)\| \Delta u \Delta s \\
\leq & \left\|\psi_{P}\left(t, t_{0}\right)\right\| \delta_{0}+\left\|\psi_{P}\left(t, t_{0}\right)\right\| \int_{t_{0}}^{T}\left\|\psi_{P}\left(t_{0}, \sigma(s)\right)\right\| \int_{t_{0}}^{s} Q(s, u)\|z(u)\| \Delta u \Delta s \\
& +\int_{T}^{t}\left\|\psi_{P}(t, \sigma(s))\right\| \int_{t_{0}}^{t_{1}} Q(s, u)\|z(u)\| \Delta u \Delta s \\
& +\int_{T}^{t}\left\|\psi_{P}(t, \sigma(s))\right\| \int_{t_{1}}^{s} Q(s, u)\|z(u)\| \Delta u \Delta s .
\end{aligned}
$$

From (6), (12), and (15), we get

$$
\int_{T}^{t}\left\|\psi_{P}(t, \sigma(s))\right\| \int_{t_{0}}^{t_{1}} Q(s, u)\|z(u)\| \Delta u \Delta s \leq \frac{(\theta-\gamma \beta) \lambda}{2 \theta \beta} .
$$

Furthermore, using (6), (10), and (14), according to

$$
\int_{T}^{t}\left\|\psi_{P}(t, \sigma(s))\right\| \int_{t_{1}}^{s} Q(s, u)\|z(u)\| \Delta u \Delta s \leq \frac{\gamma \beta \lambda}{\theta} .
$$

Then

$$
\begin{aligned}
\|z(t)\| \leq & \left\|\psi_{P}\left(t, t_{0}\right)\right\| \delta_{0}+\left\|\psi_{P}\left(t, t_{0}\right)\right\| \int_{t_{0}}^{T}\left\|\psi_{P}\left(t_{0}, \sigma(s)\right)\right\| \\
& \times \int_{t_{0}}^{s} Q(s, u)\|z(u)\| \Delta u \Delta s+\frac{(\theta+\gamma \beta) \lambda}{2 \theta} .
\end{aligned}
$$


Since $\left\|\psi_{P}\left(t, t_{0}\right)\right\| \rightarrow 0$ as $t \rightarrow \infty$, by (7) we get $\lambda+\frac{(\theta+\gamma \beta) \lambda}{2 \theta}$, and then $\lambda<\lambda$. Therefore, it is a contradiction. Thus, the zero solution of (2) is attractive. Hence, the zero solution of (2) is asymptotically stable.

\section{Exponential asymptotic stability}

Definition 5.1 The zero solution of (2) is exponentially asymptotically stable, then there exists $\eta>0$, and for every $\epsilon>0$ there exists $\delta>0$ such that, for any solution $\mathrm{z}(\mathrm{t})$ of (2), $\left\|z_{0}\right\|<\delta \Longrightarrow\|z\|<\epsilon e_{-\eta}\left(t, t_{0}\right), \forall t \in \mathbb{T}_{0}$.

Suppose that there exists $L, \eta>0$ with $-\eta \in \mathcal{R}^{+}(\mathbb{T}, \mathbb{R})$ such that

$$
\left\|\psi_{P}(t, s)\right\| \leq M e_{-v}(t, s), \quad \forall s \in\left[t_{0}, t\right]_{\mathbb{T}} .
$$

(This result is useful for proving that the zero solution of (3) is exponentially asymptotically stable.)

Theorem 5.1 Let conditions (H1) and (16) be satisfied, and there exists a positive constant $v$ such that

$$
\sup _{t \in \mathbb{T}_{0}} \int_{t_{0}}^{t} e_{-v}(s, \sigma(t)) Q(t, s) \Delta s<\frac{\eta}{L} .
$$

Then the zero solution of (2) is exponentially asymptotically stable.

Proof By using (16) $\forall t \in \mathbb{T}_{0}$ and $\left\|z_{0}\right\|<\frac{\alpha}{L}$, we get

$$
\begin{aligned}
\|z(t)\| & \leq\left\|\psi_{P}\left(t, t_{0}\right)\right\|\left\|z_{0}\right\|+\int_{t_{0}}^{t}\left\|\psi_{P}(t, \sigma(s))\right\| \int_{t_{0}}^{s} Q(s, u)\|z(u)\| \Delta u \Delta s \\
& \leq L e_{-\eta}\left(t, t_{0}\right)\left\|z_{0}\right\|+L \int_{t_{0}}^{t} e_{-\eta}(t, \sigma(s)) \int_{t_{0}}^{s} Q(s, u)\|z(u)\| \Delta u \Delta s .
\end{aligned}
$$

There exists a positive constant $a<b$ and $\epsilon$ with $-a,-\epsilon \in \mathcal{R}^{+}(\mathbb{T}, \mathbb{R})$ such that $-\eta=-a \oplus-\epsilon$, and

$$
\sup _{t \in \mathbb{T}_{0}} \int_{t_{0}}^{t} e_{-a}(s, \sigma(t)) Q(t, s) \Delta s<\frac{\epsilon}{L} .
$$

Multiplying by $e_{-a}(s, \sigma(t))$ on both sides of (18), we get

$$
\begin{aligned}
e_{-a}\left(t_{0}, t\right)\|z(t)\| \leq & L e_{-\epsilon}\left(t, t_{0}\right)\left\|z_{0}\right\|+L \int_{t_{0}}^{t} e_{-a}\left(t_{0}, \sigma(s)\right) e_{-\epsilon}(t, \sigma(s)) \\
& \times \int_{t_{0}}^{s} Q(s, u)\|z(u)\| \Delta u \Delta s \\
= & L e_{-\epsilon}\left(t, t_{0}\right)\left\|z_{0}\right\|+L \int_{t_{0}}^{t} e_{-\epsilon}(t, \sigma(s)) \\
& \times \int_{t_{0}}^{s} e_{-a}(u, \sigma(s)) Q(s, u) e_{-a}\left(t_{0}, u\right)\|z(u)\| \Delta u \Delta s .
\end{aligned}
$$


If we define $r(t)=\sup _{s \in\left[t_{0}, t\right]_{\mathbb{T}}} e_{-a}\left(t_{0}, t\right)\|z(t)\|$, consequently

$$
\begin{aligned}
e_{-a}\left(t_{0}, t\right)\|z(t)\| \leq & L e_{-\epsilon}\left(t, t_{0}\right)\left\|z_{0}\right\|+\operatorname{Lr}(t) \int_{t_{0}}^{t} e_{-\epsilon}(t, \sigma(s)) \\
& \times \int_{t_{0}}^{s} e_{-a}(u, \sigma(s)) Q(s, u) \Delta u \Delta s \\
\leq & L e_{-\epsilon}\left(t, t_{0}\right)\left\|z_{0}\right\|+\epsilon r(t) \int_{t_{0}}^{t} e_{-\epsilon}(t, \sigma(s)) \Delta s .
\end{aligned}
$$

By using [Theorem 2.39, [17]], we have

$$
e_{-a}\left(t_{0}, t\right)\|z(t)\| \leq L e_{-\epsilon}\left(t, t_{0}\right)\left\|z_{0}\right\|+\left\{1-e_{-\epsilon}\left(t, t_{0}\right)\right\} r(t)
$$

Now we consider two cases:

Case (i): If $e_{-a}\left(t_{0}, s\right)\|z(s)\| \leq e_{-a}\left(t_{0}, t\right)\|z(t)\|$ for any $s \in\left[t_{0}, t\right]_{\mathbb{T}}$. So, we get $r(t)=$ $e_{-a}\left(t_{0}, t\right)\|z(t)\|$. Then from (19) we get

$$
r(t) \leq L e_{-\epsilon}\left(t_{0}, t\right)\left\|z_{0}\right\|\left\{1-e_{-\epsilon}\left(t, t_{0}\right)\right\} r(t)
$$

Then $r(t) \leq L\left\|z_{0}\right\|, \forall t \in \mathbb{T}_{0}$. Thus, $r(t)=e_{-a}\left(t_{0}, t\right)\|z(t)\| \Longrightarrow\|z(t)\| \leq L e_{-a}\left(t_{0}, t\right)\left\|z_{0}\right\|, \forall t \in$ $\mathbb{T}_{0}$.

Case (ii): There exists $s \in\left[t_{0}, t\right]_{\mathbb{T}}$ such that $e_{-a}\left(t_{0}, s\right)\|z(s)\|>e_{-a}\left(t_{0}, t\right)\|z(t)\|$. Then there exists $t_{1} \in\left[t_{0}, t\right]_{\mathbb{T}}$ such that $r(t)=e_{-a}\left(t_{0}, t\right)\left\|z\left(t_{1}\right)\right\|$. Then from (19) we get

$$
r\left(t_{1}\right)=e_{-a}\left(t_{0}, t_{1}\right)\left\|z\left(t_{1}\right)\right\| \leq L e_{-\epsilon}\left(t_{1}, t_{0}\right)\left\|z_{0}\right\|\left\{1-e_{-\epsilon}\left(t_{0}, t_{1}\right)\right\} r\left(t_{1}\right) .
$$

Then $r\left(t_{1}\right) \leq L\left\|z_{0}\right\|, \forall t_{1} \in \mathbb{T}_{0}$. Thus, $r\left(t_{1}\right) \geq e_{-a}\left(t_{0}, t\right)\|z(t)\| \Longrightarrow\|z(t)\| \leq L e_{-a}\left(t, t_{0}\right)\left\|z_{0}\right\|$, $\forall t \in \mathbb{T}_{0}$.

Therefore, from case (i) and case (ii), the zero solution of (2) is exponentially asymptotically stable.

\section{Strong stability}

Definition 6.1 The zero solution of (2) is known as strongly stable if for all $\epsilon>0 \exists \delta>0$ such that, for any solution $\mathrm{z}(\mathrm{t})$ of (2), the inequality $t_{1} \in \mathbb{T}_{0}$ and $\left\|z\left(t_{0}\right)\right\|<\delta \Longrightarrow\|z(t)\|<$ $\epsilon, \forall t \leq t_{0} \in \mathbb{T}_{0}$.

Theorem 6.1 ([21], Theorem 4.3) Let $\psi_{P}(t, s)$ be a fundamental matrix for (3). Then the zero solution of (3) is strongly stable on $\mathrm{T}_{0}$ if and only if there exists a positive constant $G$ such that

$$
\left\|\psi_{P}\left(t, t_{0}\right) \psi_{P}^{-1}\left(s, t_{0}\right)\right\| \leq G, \quad \forall t_{0} \leq s \leq t<\infty
$$

or equivalently,

$$
\left\|\psi_{P}\left(t, t_{0}\right)\right\| \leq G \quad \text { and } \quad\left\|\psi_{P}^{-1}\left(s, t_{0}\right)\right\| \leq G, \quad \forall t \in \mathbb{T}_{0}
$$

We need the following assumptions: 
$K_{1}$ : There exist a continuous function $\varphi: \mathbb{T}_{0} \rightarrow(0, \infty)$ and constants $q_{1} \geq 1, G_{1}>0$ such that

$$
\int_{t_{0}}^{t}\left(\varphi(s)\left\|\psi_{P}\left(t, t_{0}\right) \psi_{P}^{-1}\left(s, t_{0}\right)\right\|\right)^{q_{1}} \Delta s \leq G_{1}, \quad \forall t \in \mathbb{T}_{0} .
$$

$K_{2}$ : There exist a continuous function $\varphi: \mathbb{T}_{0} \rightarrow(0, \infty)$ and constants $q_{2} \geq 1, G_{2}>0$ such that

$$
\int_{t_{0}}^{t}\left(\varphi(s)\left\|\psi_{P}\left(t, t_{0}\right) \psi_{P}^{-1}\left(s, t_{0}\right)\right\|\right)^{q_{2}} \Delta s \leq G_{2}, \quad \forall t \in \mathbb{T}_{0} .
$$

$K_{3}$ : There exist a continuous function $\varphi: \mathbb{T}_{0} \rightarrow(0, \infty)$ and constants $q_{3} \geq 1, G_{3}>0$ such that

$$
\int_{t_{0}}^{t}\left(\varphi(s)\left\|\psi_{P}\left(t, t_{0}\right) \psi_{P}^{-1}\left(s, t_{0}\right)\right\|\right)^{q_{3}} \Delta s \leq G_{3}, \quad \forall t \in \mathbb{T}_{0}
$$

$K_{4}$ : There exist a continuous function $\varphi: \mathbb{T}_{0} \rightarrow(0, \infty)$ and constants $q_{4} \geq 1, G_{4}>0$ such that

$$
\int_{t_{0}}^{t}\left(\varphi(s)\left\|\psi_{P}\left(t, t_{0}\right) \psi_{P}^{-1}\left(s, t_{0}\right)\right\|\right)^{q_{4}} \Delta s \leq G_{4}, \quad \forall t \in \mathbb{T}_{0}
$$

Theorem 6.2 Assume that the fundamental matrix $\psi_{P}\left(t, t_{0}\right)$ satisfies one of the following assumptions:

$Q_{1}: K_{1}$ and $K_{2}$ are true;

$Q_{2}: K_{1}$ and $K_{4}$ are true;

$Q_{3}: K_{2}$ and $K_{3}$ are true;

$Q_{4}: K_{3}$ and $K_{4}$ are true.

Thus, the zero solution of (3) is strongly stable on $\mathbb{T}_{0}$.

Proof . We show that $\psi_{P}\left(t, t_{0}\right)$ and $\psi_{P}^{-1}\left(t, t_{0}\right)$ are bounded on $\mathbb{T}_{0}$. First, we consider case $Q_{2}$. For this we show that $\psi_{P}\left(t, t_{0}\right)$ is bounded on $\mathbb{T}_{0}$.

Consider

$$
r(t)=\varphi^{q_{1}}(t)\left(\psi_{P}\left(t, t_{0}\right)\right)^{-q_{1}}, \quad \forall t \in \mathbb{T}_{0} .
$$

From the identity

$$
\begin{aligned}
\left(\int_{t_{0}}^{t} r(s) \Delta s\right) \psi_{P}\left(t, t_{0}\right)= & \int_{t_{0}}^{t}\left(\varphi(s) \psi_{P}\left(t, t_{0}\right) \psi_{P}^{-1}\left(s, t_{0}\right)\right) \\
& \times\left(r(s)(\varphi(s))^{-1} \psi_{P}\left(s, t_{0}\right)\right) \Delta s, \quad \forall t \in \mathbb{T}_{0} .
\end{aligned}
$$

Consequently,

$$
\begin{aligned}
\left(\int_{t_{0}}^{t} r(s) \Delta s\right)\left\|\psi_{P}\left(t, t_{0}\right)\right\| \leq & \int_{t_{0}}^{t}\left(\varphi(s)\left\|\psi_{P}\left(t, t_{0}\right) \psi_{P}^{-1}\left(s, t_{0}\right)\right\|\right) \\
& \times\left(r(s)(\varphi(s))^{-1}\left\|\psi_{P}\left(s, t_{0}\right)\right\|\right) \Delta s, \quad \forall t \in \mathbb{T}_{0} .
\end{aligned}
$$


Ayyalappagari and Bhogapurapu Advances in Difference Equations

(2021) $2021: 514$

Page 11 of 17

If $q_{1}=1$, we get $r(s)(\varphi(s))^{-1}\left\|\psi_{P}\left(s, t_{0}\right)\right\|=1$. From (20) and hypothesis $K_{1}$, we have

$$
\left(\int_{t_{0}}^{t} r(s) \Delta s\right)\left\|\psi_{P}\left(t, t_{0}\right)\right\| \leq \int_{t_{0}}^{t}\left(\varphi(s)\left\|\psi_{P}\left(t, t_{0}\right) \psi_{P}^{-1}\left(s, t_{0}\right)\right\|\right) \Delta s, \quad \forall t \in \mathbb{T}_{0} .
$$

If $q_{1}>1$, set $r_{1}=\frac{q_{1}}{q_{1}-1}$ such that $r(s)(\varphi(s))^{-1}\left\|\psi_{P}\left(s, t_{0}\right)\right\|=(r(s))^{\frac{1}{r_{1}}}$. From (20), we obtain

$$
\left(\int_{t_{0}}^{t} r(s) \Delta s\right) \varphi(t)(r(t))^{\frac{1}{q_{1}}} \leq \int_{t_{0}}^{t}\left(\varphi(s)\left\|\psi_{P}\left(t, t_{0}\right) \psi_{P}^{-1}\left(s, t_{0}\right)\right\|\right)(r(s))^{\frac{1}{r_{1}}} \Delta s, \quad \forall t \in \mathbb{T}_{0} .
$$

Using Holder's inequality [17], we obtain

$$
\begin{aligned}
\left(\int_{t_{0}}^{t} r(s) \Delta s\right) \varphi(t)(r(t))^{\frac{1}{q_{1}}} \leq & \left(\int_{t_{0}}^{t}\left(\varphi(s)\left\|\psi_{P}\left(t, t_{0}\right) \psi_{P}^{-1}\left(s, t_{0}\right)\right\|\right)^{q_{1}} \Delta s\right)^{\frac{1}{q_{1}}} \\
& \times\left(\int_{t_{0}}^{t}(r(s)) \Delta s\right)^{\frac{1}{r_{1}}}, \quad \forall t \in \mathbb{T}_{0} .
\end{aligned}
$$

Next, by using assumptions $K_{1}$, we have

$$
\begin{aligned}
& \left(\int_{t_{0}}^{t} r(s) \Delta s\right)^{\frac{1}{q_{1}}} \varphi(t)(r(t))^{\frac{1}{p_{1}}} \leq G_{1}^{\frac{1}{q_{1}}}, \quad \forall t \in \mathbb{T}_{0} . \\
& \int_{t_{0}}^{t} r(s) \Delta s\left\|\psi_{P}\left(t, t_{0}\right)\right\|^{q_{1}} \leq G_{1}, \quad \forall t \in \mathbb{T}_{0} .
\end{aligned}
$$

Then, for $q_{1} \geq 1$, the function $\left\|\psi_{P}\left(t, t_{0}\right)\right\|$ satisfies the inequality

$$
\left\|\psi_{P}\left(t, t_{0}\right)\right\|^{q_{1}} \leq G_{1}^{\frac{1}{q_{1}}}\left(\int_{t_{0}}^{t} r(s) \Delta s\right)^{\frac{-1}{q_{1}}}, \quad \forall t \in \mathbb{T}_{0} .
$$

Let $R(t)=\int_{t_{0}}^{t} r(s) \Delta s, \forall t \in \mathbb{T}_{0}$, so we obtain

$$
\left\|\psi_{P}\left(t, t_{0}\right)\right\|^{q_{1}} \leq G_{1}^{\frac{1}{q_{1}}}(R(t))^{\frac{-1}{q_{1}}} \quad \forall t \in \mathbb{T}_{0}
$$

Note $R^{\Delta}(t)=r(t) \geq G_{1}^{-1}(\varphi(t))^{q_{1}} R(t), \forall t \in \mathbb{T}_{0}$.

Consequently, there exists a constant $L_{1}$ such that

$$
\left\|\psi_{P}\left(t, t_{0}\right)\right\| \leq L_{1}, \quad \forall t \in \mathbb{T}_{0}
$$

Next, we prove that $\psi_{P}^{-1}\left(t, t_{0}\right)$ is bounded on $\mathbb{T}_{0}$.

Consider

$$
r(t)=\varphi^{p 4}(t)\left\|\psi_{P}^{-1}\left(t, t_{0}\right)\right\|^{q 4}, \quad t \in \mathbb{T}_{0} .
$$

From the identity

$$
\left(\int_{t_{0}}^{t} r(s) \Delta s\right) \psi_{P}^{-1}\left(t, t_{0}\right)=\int_{t_{0}}^{t}\left(r(s)(\varphi(t))^{-1} \psi_{P}^{-1}\left(s, t_{0}\right)\right)
$$




$$
\times\left(\varphi(s) \psi_{P}\left(s, t_{0}\right) \psi_{P}^{-1}\left(t, t_{0}\right)\right) \Delta s, \quad \forall t \in \mathbb{T}_{0}
$$

Consequently,

$$
\begin{aligned}
\left(\int_{t_{0}}^{t} r(s) \Delta s\right)\left\|\psi_{P}^{-1}\left(t, t_{0}\right)\right\| \leq & \int_{t_{0}}^{t}\left(r(s)(\varphi(t))^{-1}\left\|\psi_{P}^{-1}\left(s, t_{0}\right)\right\|\right) \\
& \times\left(\varphi(s)\left\|\psi_{P}\left(s, t_{0}\right) \psi_{P}^{-1}\left(t, t_{0}\right)\right\|\right) \Delta s, \quad \forall t \in \mathbb{T}_{0} .
\end{aligned}
$$

If $q_{4}=1$, we get $r(s)(\varphi(t))^{-1}\left\|\psi_{P}^{-1}\left(s, t_{0}\right)\right\|=1$. Using assumptions $G_{4}$, we get

$$
\left(\int_{t_{0}}^{t} r(s) \Delta s\right)\left\|\psi_{P}^{-1}\left(t, t_{0}\right)\right\| \leq \int_{t_{0}}^{t}\left(\varphi(s)\left\|\psi_{P}\left(s, t_{0}\right) \psi_{P}^{-1}\left(t, t_{0}\right)\right\|\right) \Delta s, \quad \forall t \in \mathbb{T}_{0} .
$$

If $q_{4}>1$, set $r_{4}=\frac{q_{4}}{q_{4}-1}$ such that $r(s)(\varphi(t))^{-1}\left\|\psi_{P}^{-1}\left(s, t_{0}\right)\right\|=(r(s))^{\frac{1}{r_{4}}}$. Consequently,

$$
\left(\int_{t_{0}}^{t} r(s) \Delta s\right)\left\|\psi_{P}^{-1}\left(t, t_{0}\right)\right\| \leq \int_{t_{0}}^{t}\left(\varphi(s)\left\|\psi_{P}\left(s, t_{0}\right) \psi_{P}^{-1}\left(t, t_{0}\right)\right\|\right)(r(s))^{\frac{1}{r_{4}}} \Delta s, \quad \forall t \in \mathbb{T}_{0} .
$$

Using Holder's inequality leads to

$$
\begin{aligned}
& \left(\int_{t_{0}}^{t} r(s) \Delta s\right)\left\|\psi_{P}^{-1}\left(t, t_{0}\right)\right\| \\
& \quad \leq\left(\int_{t_{0}}^{t}\left(\varphi(s)\left\|\psi_{P}\left(s, t_{0}\right) \psi_{P}^{-1}\left(t, t_{0}\right)\right\|\right)^{q_{4}} \Delta s\right)^{\frac{1}{q_{4}}}(r(s))^{\frac{1}{r_{4}}}, \quad \forall t \in \mathbb{T}_{0} .
\end{aligned}
$$

Now, by using assumption $K_{4}$, we obtain

$$
\left(\int_{t_{0}}^{t} r(s) \Delta s\right)\left\|\psi_{P}^{-1}\left(t, t_{0}\right)\right\| \leq\left(\int_{t_{0}}^{t} r(s) \Delta s\right)^{\frac{1}{r_{4}}}\left(K_{4}\right)^{\frac{1}{q_{4}}}, \quad \forall t \in \mathbb{T}_{0} .
$$

or

$$
\left(\int_{t_{0}}^{t} r(s) \Delta s\right)^{\frac{1}{q_{4}}}\left\|\psi_{P}\left(t, t_{0}\right)\right\| \leq\left(K_{4}\right)^{\frac{1}{q_{4}}}, \quad \forall t \in \mathbb{T}_{0} .
$$

Then, for $q_{4} \geq 1$, the function $\left\|\psi_{P}^{-1}\left(t, t_{0}\right)\right\|$ satisfies the inequality

$$
\left\|\psi_{P}^{-1}\left(t, t_{0}\right)\right\| \leq\left(K_{4}\right)^{\frac{1}{q_{4}}}\left(\int_{t_{0}}^{t} r(s) \Delta s\right)^{\frac{-1}{q_{4}}}, \quad \forall t \in \mathbb{T}_{0} .
$$

Let $R(t)=\int_{t_{0}}^{t} r(s) \Delta s, \forall t \in \mathbb{T}_{0}$, we obtain

$$
\left\|\psi_{P}^{-1}\left(t, t_{0}\right)\right\| \leq\left(G_{4}\right)^{\frac{1}{q_{4}}}(R(t))^{\frac{-1}{q_{4}}}, \quad \forall t \in \mathbb{T}_{0}
$$

Note $R^{\Delta}(t)=r(t) \geq G_{4}^{-1}(\varphi(t))^{q_{4}} R(t), \forall t \in \mathbb{T}_{0}$. Then there exists a constant $L_{2}$ such that

$$
\left\|\psi_{P}^{-1}\left(t, t_{0}\right)\right\| \leq L_{2}, \quad \forall t \in \mathbb{T}_{0}
$$


Hence, the result holds by using Theorem 6.1. Similarly, the theorem is valid for remaining cases $Q_{1}, Q_{3}$, or $Q_{4}$.

Theorem 6.3 Suppose that the function $G$ is continuous and satisfies the following assumption:

$$
\|G(t, s, z)-G(t, s, x)\| \leq h(t, s)\|z-x\| .
$$

For $t_{0} \leq s \leq t<\infty$ and $\forall z, x \in \mathbb{R}^{n^{2}}$, there exists a function $\beta \in \mathcal{R}^{+}\left(\mathbb{T}_{0}, \mathbb{R}\right)$ such that

$$
\sup _{t \in \mathbb{T}_{0}} \frac{1}{e_{\beta}\left(t, t_{0}\right)} \int_{t_{0}}^{t}\left\|\psi_{P}(t, \sigma(s))\right\| \int_{t_{0}}^{s} h(s, u) e_{\beta}\left(u, t_{0}\right) \Delta u \Delta s<1
$$

and

$$
\sup _{t \in \mathbb{T}_{0}} \frac{1}{e_{\beta}\left(t, t_{0}\right)}\left\|\psi_{P}\left(t, t_{0}\right)\right\|<\infty,
$$

where $h$ is an $r d$-continuous nonnegative function on $C=\left\{(t, s): t_{0} \leq s \leq t<\infty\right\}$. Then there exists a unique solution of (2).

Proof We consider the space of continuous function $Q\left(\mathbb{T}_{0} ; \mathbb{R}^{n^{2}}\right)$ with

$$
\sup _{t \in \mathbb{T}_{0}} \frac{\|z(t)\|}{e_{\beta}\left(t, t_{0}\right)}<\infty
$$

and we denote this space by $Q_{\beta}\left(\mathbb{T}_{0} ; \mathbb{R}^{n^{2}}\right)$. We couple the linear space $Q_{\beta}\left(\mathbb{T}_{0} ; \mathbb{R}^{n^{2}}\right)$ with a metric, namely

$$
C_{\beta}^{\infty}=\sup _{t \in \mathbb{T}_{0}} \frac{\|z(t)-x(t)\|}{e_{\beta}\left(t, t_{0}\right)} .
$$

It is easy to understand that $Q\left(\mathbb{T}_{0} ; \mathbb{R}^{n^{2}}\right)$ (coupled with the norm $\left(\|z\|_{\beta}^{\infty}=\sup _{t \in \mathbb{T}_{0}} \frac{\|z(t)\|}{e_{\beta}\left(t, t_{0}\right)}\right)$ is a Banach space ([15], Lemma 4.1).

Consider the operator $\mathrm{T}$ from $Q_{\beta}\left(\mathbb{T}_{0} ; \mathbb{R}^{n^{2}}\right)$ to $Q_{\beta}\left(\mathbb{T}_{0} ; \mathbb{R}^{n^{2}}\right)$, given by

$$
T z(t)=\psi_{P}\left(t, t_{0}\right) z\left(t_{0}\right)+\int_{t_{0}}^{t}\left\|\psi_{P}(t, \sigma(s))\right\| \int_{t_{0}}^{s} G(s, u, z) \Delta u \Delta s
$$

and note

$$
\begin{aligned}
\|T z\|_{\beta}^{\infty}= & \sup _{t \in \mathbb{T}} \frac{1}{e_{\beta}\left(t, t_{0}\right)}\left\|\psi_{P}\left(t, t_{0}\right) z\left(t_{0}\right)+\int_{t_{0}}^{t} \psi_{P}(t, \sigma(s)) \int_{t_{0}}^{s} G(s, u, z) \Delta u \Delta s\right\| \\
\leq & \sup _{t \in \mathbb{T}} \frac{1}{e_{\beta}\left(t, t_{0}\right)}\left(\left\|\psi_{P}\left(t, t_{0}\right) z\left(t_{0}\right)\right\|+\int_{t_{0}}^{t}\left\|\psi_{P}(t, \sigma(s)) \int_{t_{0}}^{s} G(s, u, z)\right\| \Delta u \Delta s\right) \\
\leq & \sup _{t \in \mathbb{T}} \frac{1}{e_{\beta}\left(t, t_{0}\right)}\left\|\psi_{P}\left(t, t_{0}\right)\right\|\left\|z\left(t_{0}\right)\right\|+\sup _{t \in \mathbb{T}} \frac{1}{e_{\beta}\left(t, t_{0}\right)} \\
& \times \int_{t_{0}}^{t}\left\|\psi_{P}(t, \sigma(s))\right\| \int_{t_{0}}^{s}\|G(s, u, z)\| \Delta u \Delta s .
\end{aligned}
$$


By using (21), (22), and (23), we have

$$
\begin{aligned}
\|T z\|_{\beta}^{\infty} \leq & \sup _{t \in \mathbb{T}} \frac{1}{e_{\beta}\left(t, t_{0}\right)}\left\|\psi_{P}\left(t, t_{0}\right)\right\|\left\|z\left(t_{0}\right)\right\| \\
& +\sup _{t \in \mathbb{T}} \frac{1}{e_{\beta}\left(t, t_{0}\right)} \int_{t_{0}}^{t}\left\|\psi_{P}(t, \sigma(s))\right\| \int_{t_{0}}^{s} h(s, u)\|z(u)\| \Delta u \Delta s \\
\leq & \sup _{t \in \mathbb{T}} \frac{1}{e_{\beta}\left(t, t_{0}\right)}\left\|\psi_{P}\left(t, t_{0}\right)\right\|\left\|z\left(t_{0}\right)\right\|+\sup _{t \in \mathbb{T}} \frac{1}{e_{\beta}\left(t, t_{0}\right)} \\
& \times \int_{t_{0}}^{t}\left\|\psi_{P}(t, \sigma(s))\right\| \int_{t_{0}}^{s} h(s, u) e_{\beta}\left(u, t_{0}\right) \frac{\|z(u)\|}{e_{\beta}\left(u, t_{0}\right)} \Delta u \Delta s \\
\leq & \sup _{t \in \mathbb{T}} \frac{1}{e_{\beta}\left(t, t_{0}\right)}\left\|\psi_{P}\left(t, t_{0}\right)\right\|\left\|z\left(t_{0}\right)\right\|+\|z\|_{\beta}^{\infty} \sup _{t \in \mathbb{T}} \frac{1}{e_{\beta}\left(t, t_{0}\right)} \\
& \times \int_{t_{0}}^{t}\left\|\psi_{P}(t, \sigma(s))\right\| \int_{t_{0}}^{s} h(s, u) e_{\beta}\left(u, t_{0}\right) \Delta u \Delta s .
\end{aligned}
$$

Also,

$$
\begin{aligned}
\|T z-T x\|_{\beta}^{\infty} & \leq \sup _{t \in \mathbb{T}} \frac{1}{e_{\beta}\left(t, t_{0}\right)}\left\|\int_{t_{0}}^{t} \psi_{P}(t, \sigma(s)) \int_{t_{0}}^{s} G(s, u, z)-G(s, u, x) \Delta u \Delta s\right\| \\
& \leq \sup _{t \in \mathbb{T}} \frac{1}{e_{\beta}\left(t, t_{0}\right)} \int_{t_{0}}^{t}\left\|\psi_{P}(t, \sigma(s))\right\| \int_{t_{0}}^{s}\|G(s, u, z)-G(s, u, x)\| \Delta u \Delta s \\
& \leq \sup _{t \in \mathbb{T}} \frac{1}{e_{\beta}\left(t, t_{0}\right)} \int_{t_{0}}^{t}\left\|\psi_{P}(t, \sigma(s))\right\| \int_{t_{0}}^{s} h(s, u)\|z(u)-x(u)\| \Delta u \Delta s \\
& \leq\|z-x\|_{\beta}^{\infty} \sup _{t \in \mathbb{T}} \frac{1}{e_{\beta}\left(t, t_{0}\right)} \int_{t_{0}}^{t}\left\|\psi_{P}(t, \sigma(s))\right\| \int_{t_{0}}^{s} h(s, u) e_{\beta}\left(u, t_{0}\right) \Delta u \Delta s .
\end{aligned}
$$

Hence, $\mathrm{T}$ is a contraction. Then there exists a unique solution of system (2) by the Banach fixed points theorem (22), (23).

Theorem 6.4 Suppose that the function $G$ is continuous and satisfies the following assumption:

$$
\|G(t, s, z)-G(t, s, x)\| \leq h(t, s)\|z-x\|
$$

for $t_{0} \leq s \leq t<\infty$ and $\forall z, x \in \mathbb{R}^{n^{2}}$ such that

$$
\sup _{t \in \mathbb{T}_{0}} \frac{1}{e_{\beta}\left(t, t_{0}\right)} \int_{t_{0}}^{t}\left\|\psi_{P}(t, \sigma(s))\right\| \int_{t_{0}}^{s} h(s, u) \Delta u \Delta s<1
$$

and

$$
N=\sup _{t \in \mathrm{T}_{0}}\left\|\psi_{P}\left(t, t_{0}\right)\right\|<\infty
$$

where $h$ is an rd-continuous nonnegative function on $C=\left\{(t, s): t_{0} \leq s \leq t<\infty\right\}$. Thus, the zero unique solution of (2) is strongly stable on $\mathbb{T}_{0}$. 
Proof For $t_{0} \in \mathbb{T}_{0}$, equation (24) becomes

$$
\begin{aligned}
T z(t)= & \psi_{P}\left(t, t_{0}\right) z\left(t_{0}\right)+\int_{t_{0}}^{t} \psi_{P}(t, \sigma(s)) \int_{t_{0}}^{s} G(s, u, z) \Delta u \Delta s \\
= & \psi_{P}\left(t, t_{1}\right) \psi_{P}\left(t_{1}, t_{0}\right) z\left(t_{0}\right)+\int_{t_{0}}^{t_{1}} \psi_{P}(t, \sigma(s)) \int_{t_{0}}^{s} G(s, u, z) \Delta u \Delta s \\
& +\int_{t_{1}}^{t} \psi_{P}(t, \sigma(s)) \int_{t_{0}}^{s} G(s, u, z) \Delta u \Delta s \\
= & \psi_{P}\left(t, t_{1}\right) \psi_{P}\left(t_{1}, t_{0}\right) z\left(t_{0}\right)+\int_{t_{0}}^{t_{1}} \psi_{P}\left(t, t_{1}\right) \psi_{P}\left(t_{1}, \sigma(s)\right) \int_{t_{0}}^{s} G(s, u, z) \Delta u \Delta s \\
& +\int_{t_{1}}^{t} \psi_{P}(t, \sigma(s)) \int_{t_{0}}^{s} G(s, u, z) \Delta u \Delta s \\
= & \psi_{P}\left(t, t_{1}\right) T z\left(t_{1}\right)+\int_{t_{1}}^{t} \psi_{P}(t, \sigma(s)) \int_{t_{0}}^{s} G(s, u, z) \Delta u \Delta s .
\end{aligned}
$$

By applying Theorem (6.3), we deduce that there exists a unique solution $\mathrm{z}(\mathrm{t})$ of (2) on $\mathbb{T}_{0}$ such that

$$
\begin{aligned}
\|z(t)\|= & \left\|\psi_{P}\left(t, t_{1}\right)\right\|\left\|z\left(t_{1}\right)\right\|+\int_{t_{1}}^{t}\left\|\psi_{P}(t, \sigma(s))\right\| \int_{t_{0}}^{s}\|G(s, u, z)\| \Delta u \Delta s \\
\leq & \sup _{t \in\left[t_{1}, \infty\right)_{\mathbb{T}}} \int_{t_{1}}^{t}\left\|\psi_{P}(t, \sigma(s))\right\| \int_{t_{0}}^{s}\|G(s, u, z)\| \Delta u \Delta s \\
& +\sup _{t \in\left[t_{1}, \infty\right)_{\mathbb{T}}}\left\|\psi_{P}\left(t, t_{1}\right)\right\|\left\|z\left(t_{1}\right)\right\| .
\end{aligned}
$$

Now, by using (25), we get

$$
\begin{aligned}
\|z(t)\|=\sup _{t \in \mathbb{T}_{0}}\|z(t)\| \leq & \left\|z\left(t_{1}\right)\right\| \sup _{t \in\left[t_{1}, \infty\right)_{\mathbb{T}}}\left\|\psi_{P}\left(t, t_{1}\right)\right\| \\
& +\sup _{w \in\left[t_{1}, \infty\right)_{\mathbb{T}}}\|z(w)\| \sup _{t \in\left[t_{1}, \infty\right)_{\mathbb{T}}} \int_{t_{1}}^{t}\left\|\psi_{P}(t, \sigma(s))\right\| \int_{t_{0}}^{s} h(s, u) \Delta u \Delta s
\end{aligned}
$$

for $t \leq t_{0} \in \mathbb{T}_{0}$. Let $\epsilon>0$ be an arbitrary constant, and let $\delta(\epsilon)=\frac{\epsilon(1-A)}{M}$ such that

$$
\left\|z\left(t_{1}\right)\right\|<\delta(\epsilon)
$$

where $A=\sup _{t \in \mathbb{T}_{0}} \int_{t_{1}}^{t}\left\|\psi_{P}(t, \sigma(s))\right\| \int_{t_{0}}^{s} h(s, u) \Delta u \Delta s$.

Now (28), it becomes

$$
\begin{aligned}
& \sup _{t \in\left[t_{1}, \infty\right)_{\mathbb{T}}}\|z(t)\|(1-A) \leq M\left\|z\left(t_{1}\right)\right\|, \\
& \sup _{t \in\left[t_{1}, \infty\right)_{\mathbb{T}}}\|z(t)\| \leq \epsilon .
\end{aligned}
$$

Hence we proved the zero solution of (2) is strongly stable on $\mathbb{T}_{0}$. 


\section{Conclusion}

The matrix Sylvester type systems that appear in mathematical physics and Volterra integro dynamical systems play an important role in many optimization and engineering problems. Recently, times scale calculus and dynamic equations on time scales have attracted much interest of many researchers due to their main feature of generalization and unification of continuous and discrete models. In this paper, we focus our attention on establishing the stability criteria for a nonlinear Volterra integro-dynamic matrix Sylvester system on time scales. The properties of Kronecker product of matrices are used to obtain the results. First, we convert the nonlinear Volterra integro-dynamic matrix Sylvester system on time scales into its equivalent vector dynamic system on time scales with the help of vectorization operator. Later, we obtain sufficient conditions for the stability, asymptotic stability, exponential stability, and strong stability for this system. The proposed results are valid for both continuous and discrete versions of Volterra integro-dynamic matrix Sylvester systems.

\section{Acknowledgements}

The authors would like to express their sincere thanks to the editor and anonymous reviewers for constructive comments and suggestions to improve the quality of this paper.

\section{Funding}

Not applicable.

Availability of data and materials

Data sharing not applicable to this article as no datasets were generated or analyzed during the current study.

\section{Declarations}

Ethics approval and consent to participate

Not applicable.

Consent for publication

Not applicable.

Competing interests

The authors declare that they have no competing interests.

Authors' contributions

All authors contributed equally to this article. All authors read and approved the final manuscript.

\section{Author details}

${ }^{1}$ Department of Mathematics, Koneru Lakshmaiah Education Foundation and Malla Reddy Institute of Technology and

Science, Dhulapally, Secunderabad, 500100, Telangana, India. ${ }^{2}$ Department of Mathematics, Koneru Lakshmaiah

Education Foundation, Vaddeswaram, Guntur, 522502, Andhra Pradesh, India.

\section{Publisher's Note}

Springer Nature remains neutral with regard to jurisdictional claims in published maps and institutional affiliations.

Received: 12 August 2021 Accepted: 29 October 2021 Published online: 04 December 2021

\section{References}

1. Agarwal, R.P.: Difference Equations and Inequalities Theory Methods and Applications. Marcel Bekar, New York (1992)

2. Rao, T.S., Suresh Kumar, G., Murty, M.S.N.: $\psi$-stability for nonlinear difference equations. Thai J. Math. 16, 801-815 (2018)

3. Hazarika, B., Karapinar, E., Rabbani, M.: Metric like spaces to prove existence of solution for nonlinear quadratic integral equations and numerical methods to solve it. J. Comput. Appl. Math. 328, 302-313 (2018)

4. Karapinar, E., Kumari Panda, S., Lateef, D.: A new approach to the solution of Fredholm integral equations via fixed point on extending $b$-metric spaces symmetry. Symmetry 10, 512 (2018)

5. Kumari, S., Karapinar, E., Atagana, A.: A numerical schemes and comparisons for fixed point results with applications to solution of Volterra integral equations in d-metric spaces. Alex. Eng. J. 59(2), 815-827 (2020)

6. Sevinik Adiguzel, R., Karapinar, E., Erthan, I.M.: A solution to nonlinear Volterra integro dynamic equations via fixed point theory. Filomat 33(16), 5333-5345 (2019) 
7. Alqahtani, B., Aydi, K.E., Rakocevic, V.: A solution for Volterra fractional integral equations by hybrid contractions. Mathematics 7, 694 (2019)

8. Adiguzel, R.S., Aksoy, U., Karapinar, E., Erhan, I.M.: On the solutions of fractional differential equations via Geraghty type hybrid contractions. Appl. Comput. Math. 20(2), 313-333 (2021)

9. Adiguzel, R.S., Aksoy, U., Karapinar, E., Erhan, I.M.: On the solution of a boundary value problem associated with a fractional differential equation. Math. Methods Appl. Sci. (2020). https://doi.org/10.1002/mma.6652

10. Adiguzel, R.S., Aksoy, U., Karapinar, E., Erhan, I.M.: Uniqueness of solution for higher-order nonlinear fractional differential equations with multi-point and integral boundary conditions. RACSAM 115, Article ID 155 (2021)

11. Lazreg, J.E., Abbas, S., Benchohra, M., Karapınar, E.: Impulsive Caputo-Fabrizio fractional differential equations in b-metric spaces. Open Math. 19, 363-372 (2021)

12. Kim, I.-S.: Semilinear problems involving nonlinear operators of monotone type. Res. Nonlinear Anal. 2, 25-35 (2019)

13. Duc Phuong, N., Hoang Luc, N., Long, L.D.: Modified quasi boundary value method for inverse source problem of the bi-parabolic equation. Adv. Theory Nonlinear Anal. Appl. 4(3), 132-142 (2020)

14. Ein, H.S.: Maßkettenkalkul mit anwendung auf zentrmsmannigfaltingkeiten. PhD thesis, Univ. Wurzburg (1998)

15. Hilger, S.: Analysis on measure chains a unified approach to continuous and discrete calculus. Results Math. 18 18-56 (1990)

16. Bohner, M., Peterson, A.: Dynamic Equations on Time Scales an Introduction with Applications. Birkhäuser, Boston (2001)

17. Bohner, M., Peterson, A.: Advances in Dynamic Equation on Time Scales. Birkhäuser, Boston (2003)

18. Bohner, M.: Some oscillation criteria for first order delay dynamic equations. Far East J. Appl. Math. 18(3), 289-304 (2005)

19. Tisdell, C.C., Zaidi, A.H.: Basic qualitative and quantitative results for solutions to nonlinear dynamic equations on time scales with an application to economic modelling. Nonlinear Anal. 68(11), 3504-3524 (2008)

20. Dacunha, J.J.: Stability for time varying linear dynamical systems on time scales. J. Comput. Appl. Math. 17, 381-410 (2005)

21. Choi, S., Im, D.M., Koo, N.: Stability of linear dynamic systems on time scales. J. Differ. Equ. Appl. 15, 167-183 (2009)

22. Adivar, M.: Principal matrix solutions variations of parameter for Volterra integro-dynamic equations on time scales. Glasg. Math. J. 53, 468-480 (2011)

23. Kulik, T., Tisdell, C.C.: Volterra integral equations on time scale qualitative and quantitative results with applications to initial value problem on unbounded domains. Int. J. Differ. Equ. 3(1), 103-133 (2008)

24. Appa Rao, B.V., Prasad, K.A.S.N.V.: Existence of $\psi$-bounded solutions for Sylvester matrix dynamical systems on time scales. Filomat 32(12), 4209-4219 (2018)

25. Agarwal, R.P., Aksoy, U., Karapinar, E., Erthan, I.M.: F-contraction mappings on metric like spaces in connection with integral equations on time scales. RACSAM 114), Article ID 147 (2020)

26. Dacunha, J.J.: Transition matrix and generalized matrix exponential via the Peano-Baker series. J. Differ. Equ. Appl. 11, 1245-1264 (2005)

27. Murty, M.S.N., Anjaneyulu, D., Suresh Kumar, G.: Conditioning of three-point boundary value problems associated with first order matrix Lyapunov systems. J. Nonlinear Sci. Appl. 4(2), 115-125 (2011)

28. Murty, M.S.N., Suresh Kumar, G.: On $\psi$-bounded solutions for non-homogeneous matrix Lyapunov systems on r. Electron. J. Qual. Theory Differ. Equ. 62, 1 (2009)

29. Murty, M.S.N., Suresh Kumar, G., Lakshmi, P.N., Anjaneyulu, D.: On $\psi$-instability of non-linear matrix Lyapunov systems. J. Korean Math. Soc. 45(5), 1361-1378 (2008)

30. Murty, M.S.N., Suresh Kumar, G.: On dichotomy and conditioning for two-point boundary value problems associated with first order matrix Lyapunov systems. Demonstr. Math. 42(4), 731-743 (2009)

31. Murty, M.S.N., Suresh Kumar, G., Appa Rao, B.V., Prasad, K.A.S.N.V.: On controllability of fuzzy dynamical matrix Lyapunov systems. Ann. West Univ. Timisoara Math. Comput. Sci. 2, 73-87 (2013)

32. Appa Rao, B.V., Prasad, K.A.S.N.V.: Controllability and observability of Sylvester matrix dynamical systems on time scales. Kyungpook Math. J. 56, 529-539 (2016)

\section{Submit your manuscript to a SpringerOpen ${ }^{\circ}$ journal and benefit from:}

- Convenient online submission

- Rigorous peer review

- Open access: articles freely available online

- High visibility within the field

- Retaining the copyright to your article

Submit your next manuscript at $\gg$ springeropen.com 